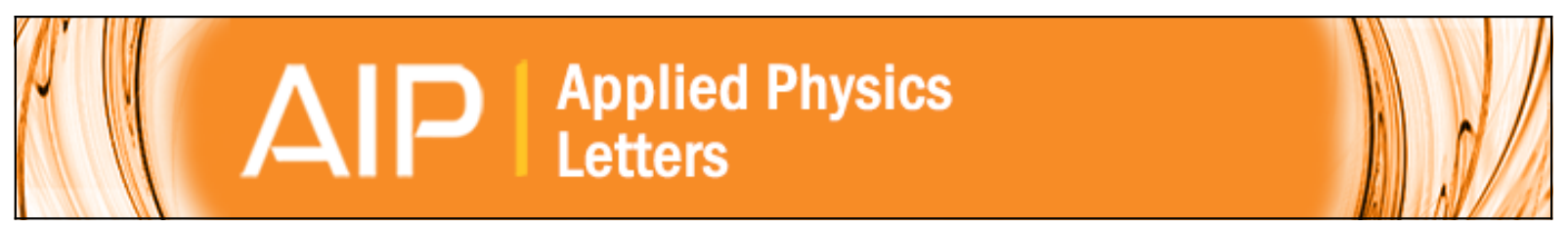

\title{
Dynamic response of shear thickening fluid under laser induced shock
}

Xianqian Wu, Fachun Zhong, Qiuyun Yin, and Chenguang Huang

Citation: Applied Physics Letters 106, 071903 (2015); doi: 10.1063/1.4913423

View online: http://dx.doi.org/10.1063/1.4913423

View Table of Contents: http://scitation.aip.org/content/aip/journal/apl/106/7?ver=pdfcov

Published by the AIP Publishing

\section{Articles you may be interested in}

Drop shape dynamics of a Newtonian drop in a non-Newtonian matrix during transient and steady shear flow

J. Rheol. 51, 261 (2007); 10.1122/1.2426973

Flow induced by a sphere settling in an aging yield-stress fluid

Phys. Fluids 18, 103101 (2006); 10.1063/1.2358090

Obtaining the shear rate profile of steady laminar tube flow of Newtonian and non-Newtonian fluids from nuclear magnetic resonance imaging and laser Doppler velocimetry data

J. Rheol. 46, 351 (2002); 10.1122/1.1446881

Laser Doppler velocimetry measurements of particle velocity fluctuations in a concentrated suspension

J. Rheol. 46, 241 (2002); 10.1122/1.1427908

An experimental study of drop deformation and breakup in extensional flow at high capillary number

Phys. Fluids 13, 1568 (2001); 10.1063/1.1358306

\section{HDDEN}

\section{Instruments for Advanced Science}

Contact Hiden Analytical for further details: w www.HidenAnalytical.com E info@hiden.co.uk CLICK TO VIEW our product catalogue

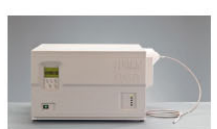

Gas Analysis

dynamic measurement of reaction gas streams catalysis and thermal analysis molecular beam studies

dissolved species probes

fermentation, environmental and ecological studies

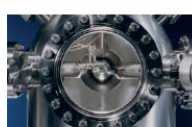

Surface Science

UHVTPD

SIMS end point detection in ion beam etch

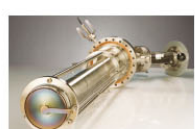

Plasma Diagnostics

- plasma source characterization etch and deposition process reaction kinetic studies , analysis of neutral and radical species

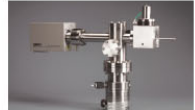

Vacuum Analysis partial pressure measurement and control of process gases reactive sputter process contro , vacuum diagnostics racum coating process monitoring 


\title{
Dynamic response of shear thickening fluid under laser induced shock
}

\author{
Xianqian Wu, ${ }^{1, a)}$ Fachun Zhong, ${ }^{2}$ Qiuyun Yin, ${ }^{1}$ and Chenguang Huang ${ }^{1}$ \\ ${ }^{1}$ Key Laboratory of Mechanics in Fluid Solid Coupling Systems, Institute of Mechanics, \\ Chinese Academy of Sciences, Beijing 100190, People's Republic of China \\ ${ }^{2}$ Institute of Chemical Materials, China Academy of Engineering Physics, Mianyang 621900, \\ People's Republic of China
}

(Received 30 January 2015; accepted 11 February 2015; published online 20 February 2015)

The dynamic response of the $57 \mathrm{vol} . / \mathrm{vol} . \%$ dense spherical silica particle-polyethylene glycol suspension at high pressure was investigated through short pulsed laser induced shock experiments. The measured back free surface velocities by a photonic Doppler velocimetry showed that the shock and the particle velocities decreased while the shock wave transmitted in the shear thickening fluid (STF), from which an equation of state for the STF was obtained. In addition, the peak stress decreased and the absorbed energy increased rapidly with increasing the thickness for a thin layer of the STF, which should be attributed to the impact-jammed behavior through compression of particle matrix, the deformation or crack of the hard-sphere particles, and the volume compression of the particles and the polyethylene glycol. (C) 2015 AIP Publishing LLC.

[http://dx.doi.org/10.1063/1.4913423]

Impact resistance and explosive protection of materials are widely studied respects for many years. Many kinds of materials such as metal foams, ${ }^{1,2}$ ceramics, ${ }^{3,4}$ and granular materials ${ }^{5-7}$ are found to show excellent high impact resistance at high pressure and high strain rates. However, the irreversible deformations are happened for these kinds of materials after a single impact. For instance, during an impact most of metal foams experience large plastic deformation and are crashed ultimately, and ceramics generate numerous cracks and are pulverized into small pieces. Recently, shear thickening fluid (STF) is observed to show high energy absorption behavior through viscous dissipation during shear deformation. ${ }^{8-11}$ More important, the energy absorption process of STF is observed to be reversible..$^{8,12,13}$ It has been regarded as a kind of advanced materials for designing composite materials and structures with high impact resistance.

In this letter, the dynamic response of a dense monodisperse suspension of hard-sphere colloidal particles at high pressure was studied. The shear thickening is a non-Newton behavior in which the viscosity increases with shear stress or shear rate while exceeding a minimum shear strain. The shear thickening behavior is observed to be result of particles rearrangement from an ordered state to a disordered state, e.g., formation of jamming particle clusters. ${ }^{8,11}$ The viscosity recovers rapidly when the shear loading is removed. ${ }^{11}$ Due to the excellent energy absorption behavior STF has founded applications in variety of fields such as damping, ${ }^{14}$ armor, ${ }^{15-18}$ to name a few. Since the impact resistance of STF is dynamic by nature, it is crucial to understand the dynamic response of the suspensions itself under dynamic loading at various pressures and strain rates. Lim et al. ${ }^{19}$ developed a split Hopkinson pressure bar method to investigate the dynamic response of viscosity fluids. Waitukaitis and Jaeger ${ }^{8}$ studied the dynamic solidification behavior of

\footnotetext{
${ }^{\text {a) }}$ Author to whom correspondence should be addressed. Electronic mail: wuxianqian@imech.ac.cn. Tel.: 86-10-8254 4256.
}

STF by directly dropping a metal rod into STF specimens. Recently, Jiang et al. ${ }^{12}$ studied the energy absorption behavior of STF using a modified SHPB setup. However, to date, studies on the impact resistance of STF have been limited to relative low pressures. The dynamic response of STF at high pressure is required to better understand the impact resistant of STF at a wide range of pressure. Here, this letter is to investigate the dynamic response of the STF at pressure up to several GPa. ${ }^{20}$ The results showed that the shock and the particle velocities, the shock pressure, and the shock energy decrease rapidly while propagating through a thin layer of STF. Saturation behaviors of the shock attenuation and energy absorption of the STF were observed.

The STF studied in this letter based on silica sphere particles with an average diameter of $300 \mathrm{~nm}$ (density $\rho_{\text {silica }}=1.950 \mathrm{~g} / \mathrm{cc}$, sound velocity $C_{\text {silica }}=5,869 \mathrm{~m} / \mathrm{s}$ ) and polyethylene glycol (density $\rho_{P E G}=1.127 \mathrm{~g} / \mathrm{cc}$, bulk modulus $K_{P E G}=3.05 \mathrm{GPa}$, sound velocity $C_{P E G}=1,650 \mathrm{~m} / \mathrm{s}$ ) with a particle volume fraction of $57 \%$. The dynamic response of the STF at high pressure was studied by laser shock experiments equipped with an in-situ shock diagnostic system for measuring back free surface velocities as depicted in Fig. 1. The STF is sealed within two aluminum plates with a size of $\Phi 25.4 \times 0.5 \mathrm{~mm}$ and a 1045 steel gasket with an inner diameter of $21.4 \mathrm{~mm}$ with the aid of silicon greases. Using a gasket with different thickness, the STF thickness in the assembled target varies from 0.12 to $5.08 \mathrm{~mm}$. The laser irradiated surface of each assembled target is glued with a $40-\mu$ m-thick aluminum foil as an absorption layer, confined firmly by a 4-mm-thick BK7 glass against the laser irradiation. A Q-switched Nd:YAG laser (near-Gaussian temporal distribution and flat spatial distribution) of $2.5 \mathrm{~J}$ per shot with a FWHM of about $10 \mathrm{~ns}$ is utilized. The focused laser diameter is $3.6 \mathrm{~mm}$ corresponding to a laser power density of $2.43 \mathrm{GW} / \mathrm{cm}^{2}$, leading to a shock pressure of $4.62 \mathrm{GPa}^{20} \mathrm{~A}$ photonic Doppler velocimetry (PDV) $)^{21-24}$ is used to capture the particle velocities during laser shocked. As shown in Fig. 2, while the shock wave propagates in the assembled 

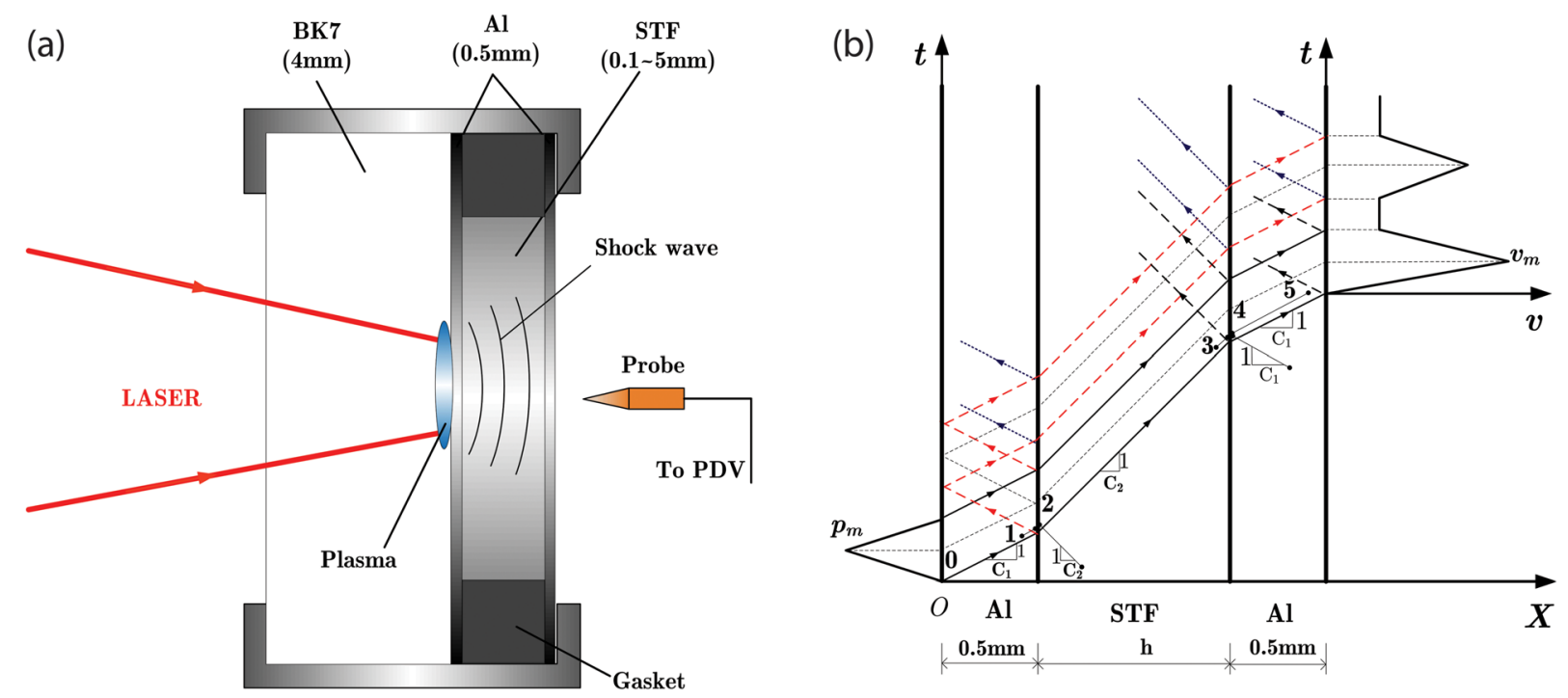

FIG. 1. Method for investigating the dynamic response of the STF. (a) Schematic of the laser induced shock experiments for the STF sealed inside two aluminum plates and a steel gasket. (b) The characteristic analysis method for analyzing the attenuation of the shock wave based on the back free surface velocities.

target, a part of the wave will reflect due to the impedance mismatch of the aluminum plates and the STF, and some rest of the shock wave will ultimately go through the assembled target and arrive at the back free surface of the right aluminum plate, causing the first peak velocity $v_{m}$. The consequential peak velocities, which are separated from the first peak, will also arrive due to the multireflection of shock wave in the assembled target. Note that only the elastic waves of the aluminum plates denoted by a wave speed $C_{1}$ and a constant wave speed of the STF denoted by $C_{2}$ are given in Fig. 2 to clearly show the analysis method. In the actual analysis, the plastic wave speed is used while the stress exceeds the Hugoniot elastic limit (HEL) of 2024 aluminum material that equals to 0.55 $\mathrm{GPa}^{25}$ Moreover, the wave speed of the STF, $C_{2}$, will be

(a)

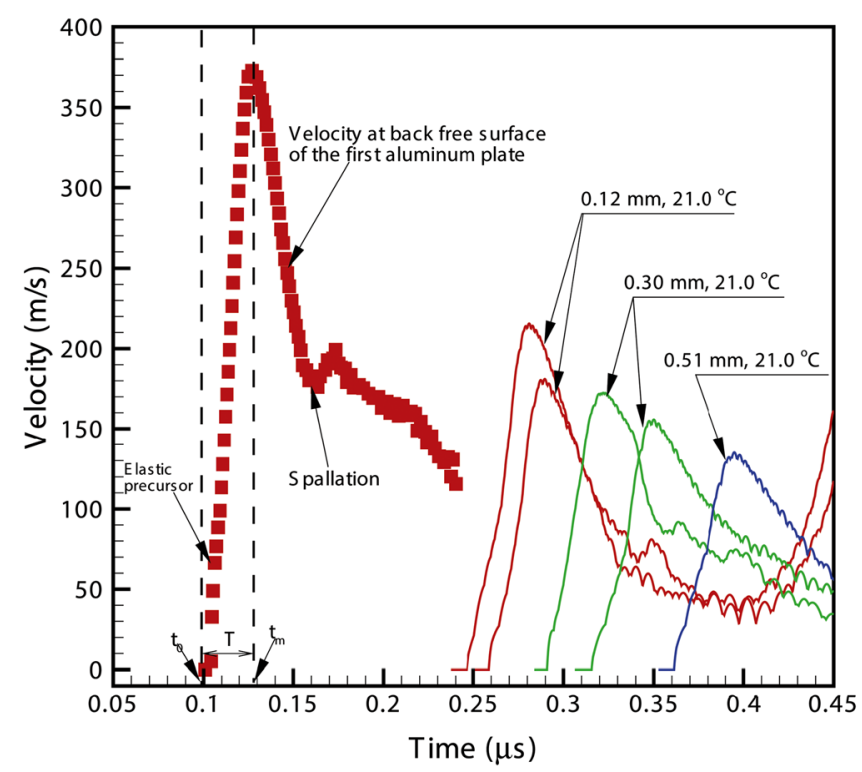

calculated according to the PDV results. A characteristic analysis ${ }^{26}$ was used to analyze the dynamic response of the STF with the first velocity peak value.

The experimental and the analysis procedure are as follows. First, the back free surface velocity of the left aluminum plate, denoted by $v_{\text {free } 1}$, is measured directly without the STF specimen and the right aluminum plate. The stress at the left surface of the STF specimen (point 2) could be calculated

$$
\sigma_{2}=-\frac{(\rho c)_{S T F} \cdot(\rho c)_{A l}}{(\rho c)_{A l}+(\rho c)_{S T F}} v_{f r e e 1}
$$

where $(\rho c)_{S T F}$ and $(\rho c)_{A l}$ are the shock impedances of the STF and the 2024 aluminum, respectively.

(b)

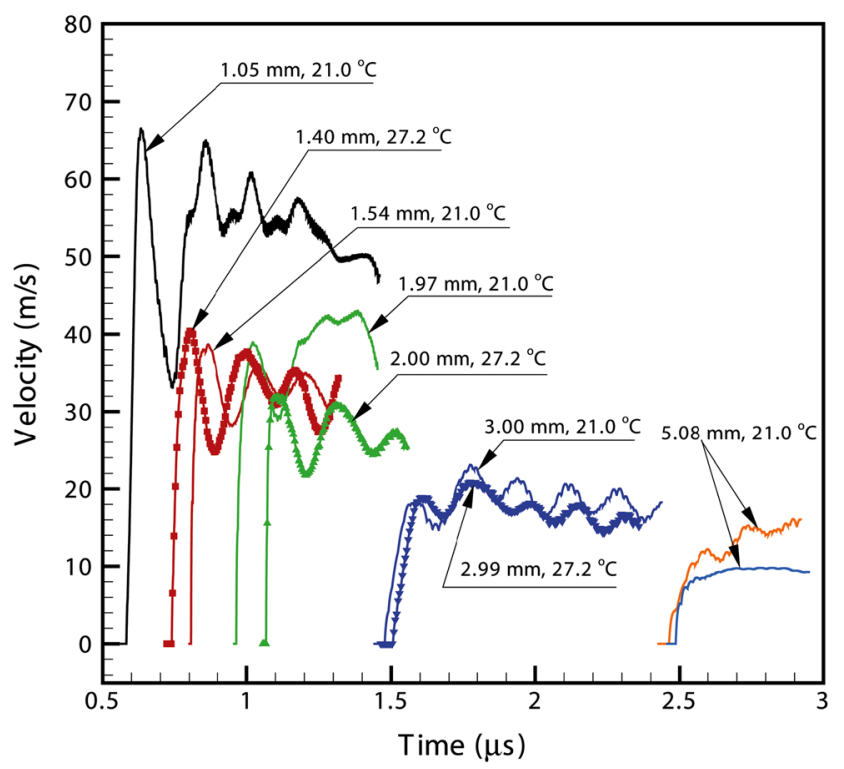

FIG. 2. (a) The measured particle velocity profile at the back free surface of the left aluminum plate, and the particle velocities at the back free surface of the right aluminum plate for a thin layer of STF varying from 0.12 to $0.51 \mathrm{~mm}$ at $21.0^{\circ} \mathrm{C}$ are also depicted. (b) The measured particle velocities at the back free surface of the right aluminum plate for a relative thick layer of the STF at $21.0^{\circ} \mathrm{C}$ and $27.2^{\circ} \mathrm{C}$. 
Then the back free surface velocity of the assembled target, denoted by $v_{\text {free } 2}$, is measured. An empirical attenuation law in 2024 aluminum material ${ }^{20}$ is undertaken to calculate the particle velocity at the point 4 , denoted by $v_{4}$. The stress at the right surface (point 3 ) could be obtained

$$
\sigma_{3}=-\frac{(\rho c)_{A l}+(\rho c)_{S T F}}{2} v_{4}
$$

In the experiments, the laser power densities were kept almost constant to ensure the same $\sigma_{2}$ for each experiment, whereas different $\sigma_{3}$ were obtained by varying the STF thickness from 0.12 to $5.08 \mathrm{~mm}$. Average shock velocities, $C_{S T F}$, within a small thickness increment of the STF and the shock attenuation could also be analyzed according to the arriving time of the PDV signals. Moreover, the absorbed energy by the STF could also be determined as

$$
\begin{aligned}
E_{S T F} & =E_{\text {Left }}-E_{\text {Right }} \\
& =\frac{A_{\text {shock }}}{(\rho c)_{\text {STF }}} \int_{0}^{T_{1}} \sigma_{2}^{2}(t) d t-\frac{A_{\text {shock }}}{(\rho c)_{\text {STF }}} \int_{0}^{T_{2}} \sigma_{3}^{2}(t) d t,
\end{aligned}
$$

where $A_{\text {shock }}$ is supposed to be the laser shocked area, and $T_{1}$ and $T_{2}$ are the durations of $\sigma_{2}$ and $\sigma_{3}$, respectively.

The measured $v_{\text {free } 1}$ is plotted in Fig. 2(a). The elastic precursor was not obvious. The particle velocity increased rapidly to a peak value of about $372.0 \mathrm{~m} / \mathrm{s}$ at $130 \mathrm{~ns}$, corresponding to a stress $\sigma_{1}$ of $3.31 \mathrm{GPa},{ }^{25}$ which is consistent with the result from a previous developed model. ${ }^{20}$ Then it decreased to $179.9 \mathrm{~m} / \mathrm{s}$ at $160 \mathrm{~ns}$ and increased a little again after that, indicating the spallation of the aluminum material. Since the spallation may occur during the laser shock, the arising duration, $T$, was taken to calculate the energy absorption of the STF in the following analysis.

The measured $v_{\text {free } 2}$ for various thicknesses and temperatures of the STF are shown in Figs. 2(a) and 2(b). The first peak particle velocity decreased rapidly from about $207 \mathrm{~m} / \mathrm{s}$ to about $38 \mathrm{~m} / \mathrm{s}$ with increasing the STF thickness from 0.12 to $1.5 \mathrm{~mm}$. The small difference of particle velocity for the same STF thickness experiments was ascribed to the uncontrolled elastic deformation of the steel gasket while clamping the assembled target. The first peak velocity decreased relative slowly with increasing the STF thickness while exceeding $1.5 \mathrm{~mm}$. Moreover, as the closed test temperatures were undertaken, the results of $21.0^{\circ} \mathrm{C}$ and $27.2^{\circ} \mathrm{C}$ did not show obvious difference beside the tests with a thickness of about $2 \mathrm{~mm}$. A relative high peak velocity was observed for the thickness $1.97 \mathrm{~mm}$ at $21.0^{\circ} \mathrm{C}$ should be ascribed to the elastic deformation of the steel gasket while preparing the assembled target.

Based on the PDV results, the average shock velocities along the thickness of the STF is calculated and plotted in Fig. 3(a). The average shock velocities decreased exponentially with increasing the STF thickness. Suppose that the STF has a similar Grüneisen equation of state (EOS) as $U_{s}=C_{0}+S U_{p},{ }^{25}$ the parameters $C_{0}$ and $S$ are calculated to be $2.05 \mathrm{~mm} / \mu$ s and 5.324 , respectively, by fitting the relationship between the average shock velocities and the first peak velocities as depicted in Fig. 3(a). The stress attenuation is given in Fig. 3(b). The peak stress decreased exponentially from $1.73 \mathrm{GPa}$ to about $68 \mathrm{MPa}$ with increasing the STF thickness. The corresponding energy absorption is shown in Fig. 3(b). The equivalent energy absorption behavior of the STF with a 62 vol./vol. \% particle volume fraction at pressures ranging from $36 \mathrm{MPa}$ to $48 \mathrm{MPa}$ was also depicted in Fig. 3(b), in which the average absorbed energy per unit volume from Jiang et al. ${ }^{12}$ was used to calculate the equivalent absorbed energy with different STF thickness. When compared to the energy absorption behavior at relative low pressure, the absorbed energy increased rapidly with increasing the STF thickness for a layer of STF less than $1 \mathrm{~mm}$. The saturation of stress attenuation and the energy absorption were observed while the thickness of the STF exceeded $2 \mathrm{~mm}$.

The fast increase of the energy absorption in a thin STF layer should be ascribed to three reasons. One is the (a)

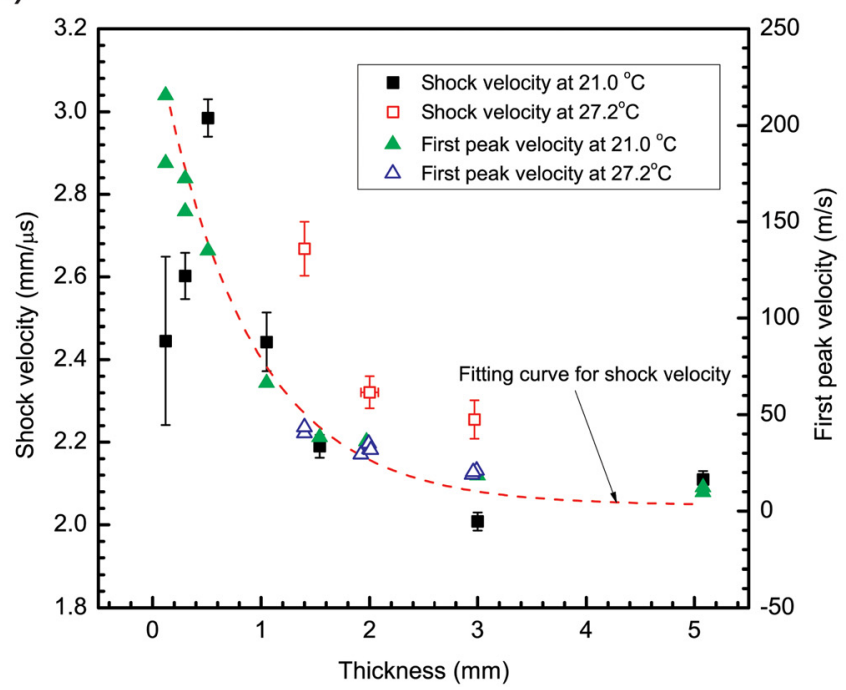

(b)

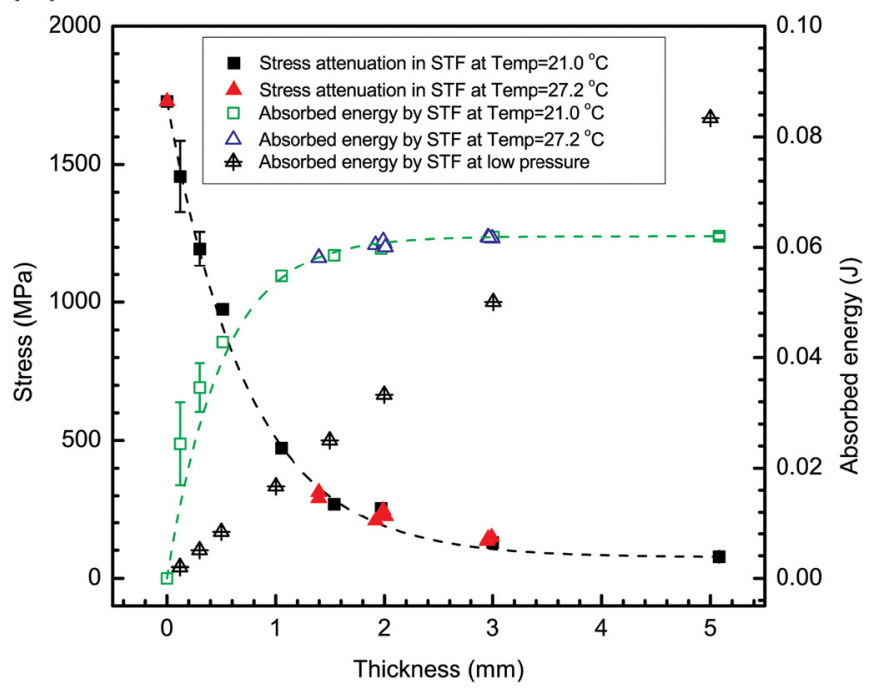

FIG. 3. (a) The average shock velocities along the thickness of the STF. (b) The stress attenuation and the corresponding energy absorption in the STF. Different shock impedance along the thickness of the STF is used in the analysis. 
impact induced jamming behavior of the STF under shock induced shock. The study by Waitukaitis and Jaeger ${ }^{8}$ showed that the particles inside the suspension were forced across the jamming transient and an impact-jammed solid through the compression of the particle matrix was rapidly growing while a rod impacts the STF directly. The impact induced jamming behavior could also occur during laser shock, leading to the energy dissipation by overcoming the short-range hydrodynamic lubrication force between the particles. The second might be the deformation or crack of hard-sphere particles while collision after the formation of the jamming. The third should be the volume compressibility of the hard-sphere particles and the polyethylene glycol at high pressure, ${ }^{25}$ leading to the further energy dissipation.

After the shock wave transmits a certain distance in the STF, the shock pressure and the particle velocity decrease to relative low values. The energy dissipation by overcoming the short-range hydrodynamic lubrication force between the particles becomes much slowly. Also, the deformation or crack of the hard-sphere particles as well as the volume compressibility of the particles and the polyethylene glycol could be neglected. In addition, the previous studies $8,19,27-30$ showed that critical conditions were required to occur the shear thickening in STF. A minimum strain might be not obtained in several tens of nanoseconds to occur the shear thickening behavior when the particle velocity decays to a relative low value, leading to the saturations of the stress attenuation and the energy absorption in the STF. Note that the critical saturation thickness of the STF should be depended on the amplitude of the shock wave. Dimensional analysis ${ }^{31}$ will be performed to investigate the dynamic response of the STF in future.

In summary, the dynamic response of the STF at high pressure was investigated by laser shock experiments. The results showed that the particle and the shock velocities decreased rapidly while the shock wave transmitted in the STF, from which an EOS for the STF was obtained. In addition, the stress attenuation and the energy absorption of the STF were obtained. The saturations of the stress attenuation and the energy absorption were observed. The fast increase of the energy absorption in a thin layer of the STF should be attributed to the impact-jammed behavior through compression of particle matrix, the deformation or crack of the hard-sphere particles, and the volume compression of the particles and the polyethylene glycol.
The authors acknowledge financial support from National Natural Science Foundation of China (Grant Nos. 11332011 and 11402277).

${ }^{1}$ D. Karagiozova, G. Langdon, and G. Nurick, Int. J. Solids Struct. 49(19), 2763-2777 (2012).

${ }^{2}$ S. L. Lopatnikov, B. A. Gama, M. Jahirul Haque, C. Krauthauser, J. W. Gillespie, Jr., M. Guden, and I. W. Hall, Compos. Struct. 61(1), 61-71 (2003).

${ }^{3}$ P. Lundberg, R. Renström, and B. Lundberg, Int. J. Impact Eng. 24(3), 259-275 (2000).

${ }^{4}$ C. G. Paalvast, M. L. Verbruggen, and L. B. Vogelesang, Google patents EP0237095B1 (1989).

${ }^{5}$ S. Antonyuk, S. Heinrich, J. Tomas, N. G. Deen, M. S. van Buijtenen, and J. Kuipers, Granular Matter 12(1), 15-47 (2010).

${ }^{6}$ C. Daraio, V. Nesterenko, E. Herbold, and S. Jin, Phys. Rev. Lett. 96(5), 058002 (2006).

${ }^{7}$ R. M. Nedderman, Statics and Kinematics of Granular Materials (Cambridge University Press, 2005).

${ }^{8}$ S. R. Waitukaitis and H. M. Jaeger, Nature 487(7406), 205-209 (2012).

${ }^{9}$ H. Laun, R. Bung, S. Hess, W. Loose, O. Hess, K. Hahn, E. Hädicke, R. Hingmann, F. Schmidt, and P. Lindner, J. Rheol. 36(4), 743-787 (1992).

${ }^{10}$ M. C. Newstein, H. Wang, N. P. Balsara, A. A. Lefebvre, Y. Shnidman, H. Watanabe, K. Osaki, T. Shikata, H. Niwa, and Y. Morishima, J. Chem. Phys. 111(10), 4827-4838 (1999).

${ }^{11}$ H. A. Barnes, J. Rheol. 33(2), 329-366 (1989).

${ }^{12}$ W. Jiang, X. Gong, S. Xuan, W. Jiang, F. Ye, X. Li, and T. Liu, Appl. Phys. Lett. 102, 101901 (2013).

${ }^{13}$ J. Bender and N. J. Wagner, J. Rheol. 40(5), 899-916 (1996).

${ }^{14}$ R. Helber, F. Doncker, and R. Bung, J. Sound Vib. 138(1), 47-57 (1990).

${ }^{15}$ Y. S. Lee, E. D. Wetzel, and N. J. Wagner, J. Mater. Sci. 38(13), 2825-2833 (2003).

${ }^{16}$ V. B. C. Tan, T. E. Tay, and W. K. Teo, Int. J. Solids Struct. 42(5-6), 1561-1576 (2005).

${ }^{17}$ M. J. Decker, C. J. Halbach, C. H. Nam, N. J. Wagner, and E. D. Wetzel, Compos. Sci. Technol. 67(3-4), 565-578 (2007).

${ }^{18}$ E. V. Lomakin, P. A. Mossakovsky, A. M. Bragov, A. K. Lomunov, A. Y. Konstantinov, M. E. Kolotnikov, F. K. Antonov, and M. S. Vakshtein, Arch. Appl. Mech. 81(12), 2007-2020 (2011).

${ }^{19}$ A. Lim, S. Lopatnikov, N. Wagner, and J. Gillespie, Jr., Rheol. Acta 49(8), 879-890 (2010).

${ }^{20}$ X. Wu, Z. Duan, H. Song, Y. Wei, X. Wang, and C. Huang, J. Appl. Phys. 110(5), 053112 (2011).

${ }^{21}$ H. Amarchinta, R. Grandhi, K. Langer, and D. Stargel, Modell. Simul. Mater. Sci. Eng. 17, 015010 (2009).

${ }^{22}$ D. Dolan, Rev. Sci. Instrum. 81, 053905 (2010).

${ }^{23}$ B. Jensen, D. Holtkamp, P. Rigg, and D. Dolan, J. Appl. Phys. 101, 013523 (2007).

${ }^{24}$ O. Strand, D. Goosman, C. Martinez, T. Whitworth, and W. Kuhlow, Rev. Sci. Instrum. 77, 083108 (2006).

${ }^{25}$ M. A. Meyers, Dynamic Behavior of Materials (Wiley-Interscience, 1994).

${ }^{26} \mathrm{~L}$. Wang, Foundations of Stress Waves (Elsevier, 2011).

${ }^{27}$ J. W. Bender and N. J. Wagner, J. Colloid Interface Sci. 172(1), 171-184 (1995).

${ }^{28}$ R. Hoffman, J. Colloid Interface Sci. 46(3), 491-506 (1974).

${ }^{29}$ B. J. Maranzano and N. J. Wagner, J. Rheol. 45(5), 1205-1222 (2001).

${ }^{30}$ B. J. Maranzano and N. J. Wagner, J. Chem. Phys. 114(23), 10514-10527 (2001).

${ }^{31}$ Q. M. Tan, Dimensional Analysis: With Case Studies in Mechanics (Springer Verlag, Beijing, 2011). 